\title{
Los entornos virtuales de simulación de la realidad, espacios vistos como ejes que permiten situar el aprendizaje dentro de un contexto institucionalizado de educación..
}

\author{
Germán Amaya Franky \\ amayag@usal.es \\ Universidad de Salamanca
}

\section{El conocimiento está situado}

"El conocimiento está situado, siendo en parte un producto de la actividad, del contexto y de la cultura en la cual se desarrolla y se utiliza" (Brown, J. 1989, p. 33-42).

La idea de concebir el aprendizaje como un producto de la actividad situada, nace de los aportes legados por autores tales como Lev Semionovitch Vygotsky (psicólogo ruso de descendencia judía). Destacado como uno de los pensadores que han resaltado el contexto como espacio de interacción y proveedor de oportunidades para la construcción consciente del conocimiento,

Cabe destacar que mucho antes, otros autores han tratado aspectos similares sobre este tema; es el caso de Hegel quien dicta en Jena (1804) un tratado filosófico en el que exalta la interacción del hombre con su entorno desde dos puntos altamente significativos, la naturaleza y el espíritu. Sus ideas tratan aspectos que permiten divisar la pertinencia del entorno y sus posibilidades en la construcción del conocimiento. Gran muestra de esto es que resalta el lenguaje como la primera categoría bajo la que el espíritu encuentra su exteriorización.

Así mismo, cabe hacer referencia a las ideas de Marx, quien exalta el trabajo como eje fundamental en el desarrollo del ser. Cabe destacar que tanto Hegel como Marx, denominan trabajo a un conglomerado de acciones que colman las necesidades que presentan los seres desde su naturaleza espiritual.

“Así el trabajo rompe el dictado del deseo inmediato y suspende, por así decirlo, el proceso de satisfacción de las necesidades. Y de la misma manera que el medio lo eran allí los símbolos lingüísticos, aquí lo son los instrumentos, en los que quedan sedimentadas las experiencias generalizadas que los que trabajan hacen con sus objetos" Habermas, J. (1986), p. 11.

Es pertinente destacar que el legado cultural se perfila en estas ideas como un elemento imprescindible que garantiza la evolución del hombre y de su conocimiento. Por consiguiente, los artefactos pasan a ser almacén de las experiencias y de las conciencias adquiridas en la interacción.

Por otro lado, James Jerónimo Gibson en sus escritos, hace alusión al término "affordances, concepto clave en Gibson que puede traducirse como oportunidades ambientales, es decir, las posibilidades que ofrece un entorno o un objeto para actuar sobre él" (Dursteler, J. 2002). Desde esta perspectiva, las propiedades que un ambiente ofrece son percibidas por el individuo como elementos significativos que orientan la acción. Al respecto, se puede destacar en los aportes de Gibson, que tanto el ambiente como el entorno ambiental son tenidos en cuenta como elementos en la toma de decisiones y en la orientación de la acción.

Pero fue en 1989 cuando el concepto de aprendizaje situado toma forma y nombre, gracias a un 
escrito presentado por Brown, J. ; Collins, A. \& Duguid, P., quienes luego de anunciar el abandono del paradigma del procesamiento de la información, deciden sustituirlo por otro denominado aprendizaje situado. Los autores toman esta determinación, luego de descubrir que la actividad y las situaciones son integrales a la cognición y al aprender, discuten la naturaleza situacional del conocimiento y la localizan como un "producto de la actividad, del contexto y la cultura en la cual se desarrolla y utiliza” (Streibel, M. 1989, 215-234).

En este artículo Brown et al, defienden el hecho de que la actividad y las situaciones son integrales a la cognición y al aprender, para lo cual citan un trabajo de Molinero y Gildea, publicado en 1987. En este escrito, se describe cómo se produce el aprendizaje de palabras desde la descontextualización. Esto es, eventos realizados al interior de los entornos instucionalizados de aprendizaje, como es el caso del uso de diccionarios u oraciones ejemplificadas, fue comparado con el aprendizaje de palabras en el contexto ordinario. El resultado de esta investigación demostró que este segundo método es mucho más práctico y rápido, ya que las personas hablando, escuchando y usando su vocabulario en el medio donde este encuentra su significado (contexto real), aprenden un promedio de 5.000 palabras por año; por el contrario, las personas dedicadas al aprendizaje desde el diccionario o actividades descontextualizadas, su aprendizaje es generalmente lento y fracasado, un máximo de 200 palabras por año. Como otro aporte, esta investigación demostró que mucho de lo que se enseña resulta ser poco útil en la práctica.

"El aprender de diccionarios, como cualquier método que intente enseñar conceptos abstractos independientes de situaciones auténticas, pasa por alto la manera como la comprensión se desarrolla con el uso continuado y situado. Este desarrollo, que implica negociaciones sociales complejas, no se cristaliza en una definición categórica. Porque es dependiente de situaciones y negociaciones, el significado de una palabra no puede, en principio, ser capturado por una definición, incluso cuando la definición es apoyada por un par de oraciones ejemplares. Todo el conocimiento es como la lengua, sus componentes son un producto de la actividad y las situaciones en las cuales se producen" (Brown, J.; Collins, A. \& Duguid, P. 1989, p. 33-42).

Desde otro aporte, Lozares, C. (2000) define la cognición situada como el producto de la interacción entre agentes y elementos del entorno; donde la utilización y adecuación de las herramientas con el medio circundante se tornan como premisas particulares en la construcción del conocimiento. Por consiguiente, para el autor "la acción o actividad situada ha de verse como interacción con los artefactos e instrumentos bajo las circunstancias sociales que los envuelven y no sólo como interacción entre sujetos sociales" (Lozares, C. 2000). A su vez, Lozares enfatiza la necesidad de tener presente en cualquier concepción de cognición situada, la integración de los elementos cognoscitivos, la praxis, los objetos, herramientas psicológicas y los demás elementos integrantes del contexto. Así mismo, destaca que el aprendizaje es un producto de la actividad, "porque las actividades son integrales a la cognición y al aprender" (Ibidem).

Al respecto, Borras, I. (1999) exalta la reciprocidad existente entre aprendizaje y actividad, esto es, "de acuerdo con esta teoría (cognición situada), el conocimiento es una relación activa entre un agente y el entorno, y el aprendizaje ocurre cuando el aprendiz está activamente envuelto en un contexto institucional complejo y realístico" (Borras, I. 1999). Así pues, Borras corrobora nuestra postura: el aprendizaje es producto de la inmersión de los sentidos en el contexto, no de la retención memorística y descontextulizada. La autora presenta el carácter situado del aprendizaje desde una postura ecologista [1], donde la relación entre pensamiento, entorno y el medio circundante, son elementos esenciales en la construcción del conocimiento.

Otro de los aportes a la concepción sobre la cognición situada, es tomado de dos autores que han dedicado gran parte de sus investigaciones al análisis de este tema: Lave, J \& Wenger, E.; es claro apreciar en sus contribuciones las ideas de Vygotsky, desde donde se ha tomado la cultura como agente acumulador de "sistemas simbólicos socialmente creados, donde se encuentran los organizadores de la experiencia” (Rodríguez, M. \& García, M. 2003, 136-144). 
"Para estos autores [Lave y Wenger], el aprendizaje situado es un aspecto inseparable e integral de cualquier práctica social. La noción de aprendizaje situado indica precisamente el carácter contextualizado del aprendizaje que no se reduce a las nociones convencionales de aprendizaje in situ o aprendizaje haciendo, sino a la participación del aprendiz en una comunidad de práctica; esto es, en un contexto cultural, social, de relaciones, de donde se obtiene los saberes necesarios para transformarla y transformarse" (Ibidem).

Creyendo haber aclarado el significado de la cognición situada, se hace pertinente concluir que este marco de inteligibilidad cognitiva se especializa en indagar cómo el conocimiento se adquiere en dependencia de un contexto situacional y cómo influyen los elementos de éste en la construcción de un aprendizaje significativo. Del mismo modo, se exalta la participación, la interacción y el compromiso del aprendiz dentro de una realidad, como características esenciales en la construcción consciente del conocimiento.

\section{Elementos del aprendizaje situado}

Con el ánimo de resaltar algunos aspectos que son elementos esenciales en toda concepción de aprendizaje bajo las premisas de la cognición situada, creemos que en primer lugar se encuentra el posibilitar un ambiente de aprendizaje realista.

"El conocimiento se construye en las interacciones del principiante con el ambiente. Aunque la duplicación exacta es a menudo imposible, la réplica cercana a un contexto verdadero del mundo mejora el aprendizaje (...). La cognición situada se basa sobre el concepto que el conocimiento es contextual y situado e influido por la actividad, el contexto y la cultura en la cual se utiliza" (Clancey, W. 1995, p. 49-70).

El posibilitar un entorno de realidad, permite a los aprendices generar un pensamiento consciente y ajustado a las necesidades situacionales. Pero así mismo, otros elementos se tornan primordiales en este marco de inteligibilidad, para el caso, un entorno de aprendizaje situado debe por lo menos posibilitar al aprendiz los siguientes elementos: La interacción dentro de un contexto socio-cultural, la interacción con un evento que se torne común para dicho contexto social, las posibilidades situacionales que le permitan generar actividad, unos recursos contextuales que le permitan la acción y la toma de decisiones. Todo lo anterior, con la finalidad de permitir al individuo ser actor de su propia construcción, desde la participación, el contacto directo con la situación para su interpretación, la negociación de significados, la clasificación y aprehensión del contenido relevante, la interacción colaborativa, la solución de problemas emergentes, la asistencia significativa de un experto (andamiaje significativo), y dentro de todo esto, asegurar la pertenencia al interior de una comunidad de práctica. Cabe resaltar que una comunidad de práctica es un entorno social que comparte tareas y actividades comunes dentro de un mismo objetivo; así como un legado de entendimiento cultural, social y situacional. (Wenger, E. 2001).

Teniendo presente lo dicho hasta este momento, surge un interrogante que nos motiva a reflexionar, esto es, sabiendo que al interior de los entornos institucionalizados de aprendizaje, es decir, en el entorno de clase tradicional, no es posible en todo momento contar con eventos que permitan el desarrollo de actividades reales y contextualizadas, ¿cómo se podría diseñar actividades pedagógicas que integren los elementos que propone la cognición situada?

La respuesta a este interrogante ha motivado desde siempre a profesionales de la educación a indagar en variados recursos que permitan ofrecer a los educandos un entorno significativo de realidad. Hoy, gracias a los adelantos en materia de nuevas tecnologías de la información y la comunicación, surgen alternativas que pretenden generar una solución a dicho problema. Al respecto, las herramientas infovirtuales permiten simular y manipular la realidad, a tal escala que están siendo adoptadas en el sector de la educación como un suplemento a dicha realidad. Sin embargo, se hace necesaria una interpretación de estos espacios con miras a comprender su verdadera potencialidad. En otras palabras, es pertinente interpretar los entornos de simulación computarizada como espacios que 
pueden ser utilizados para generar entornos situacionales de realidad social, cultural, situacional y de actividad.

\section{La simulación computarizada de la realidad}

En este aparte se pretende esbozar una interpretación de los entornos computarizados de simulación desde el marco de inteligibilidad anteriormente descrito. Por lo tanto, se hace imprescindible unificar algunos conceptos que son la base de su interpretación.

Los entornos de simulación computarizada se pueden definir como un "acontecimiento estructurado que encarna relaciones causales entre el elemento y evento que representa una situación del mundo real" (Duchastel 1990, citado en Martí, E.(1992). Desde otro aporte, Delval, J. (1986) define la simulación como la reproducción de una situación o un fenómeno que se presenta generalmente simplificado y, que a su vez, permite la manipulación de sus variables intervinientes. Entonces, las simulaciones deben constituir un "modelo de situación o de fenómeno, en el que aparecen los aspectos que consideramos importantes para nuestro propósito, despreciando así los que son secundarios o accesorios" (Delval, J. 1986, p.154). Así pues, podríamos decir que una simulación representa el desarrollo de la realidad en forma parcial, es decir, procura representar, en parte, el funcionamiento de un sistema real.

Desde lo anterior, la simulación computarizada es la representación digital de una parte de la realidad; expresada mediante un sistema info-virtual manipulable que puede ser natural, artificial o imaginario. Partiendo de esta definición, vemos pertinente unificar dos términos que se tornan imprescindibles para encontrar sentido a lo que se está diciendo, estas son: sistema y modelo.

\section{Sistema}

Un sistema es, "una colección o conjunto de elementos o normas que ordenados cumplen un fin particular"(Martínez, S. \& Requena, A. 1986, p. 34). En palabras de Shannon, R. (1988) es un conjunto de objetos o ideas que están interrelacionados entre sí como una unidad para la consecución de un fin lógico. También lo define, como la porción del universo que será objeto de la simulación.

Las anteriores definiciones hacen entrever que la característica esencial de un sistema, es la interrelación existente entre cada una de sus partes con relación a ciertas reglas enmarcadas dentro de un objetivo. En consecuencia, un sistema puede realizar una función que no es realizable por sus componentes en forma individual.

\section{Modelo}

El modelo es un objeto o evento que puede ser empleado por un individuo para indagar y responder aspectos de su interés con relación al objeto o evento real. Para Law A. \& Kelton W., (1991), un objeto $X$ es un modelo del objeto $Y$ para el observador $Z$, si $Z$ puede emplear a $X$ para responder cuestiones que le interesan acerca de $Y$. En tal sentido, un modelo de simulación es "el conjunto de hipótesis acerca del funcionamiento del sistema expresado como relaciones matemáticas y/o lógicas entre los elementos del sistema"(García, C. \& Villatoro, F., p .1).

Por consiguiente, las simulaciones se componen de modelos que representan una parte o la totalidad de un sistema. Así pues, las simulaciones se caracterizan por estar compuestas de las siguientes partes:

1. Un modelo: corresponde a la parte simbólica de la simulación, generalmente es un conjunto de ecuaciones o reglas que pertenecen a una lógica matemática o estadística.

2. El evaluador: es el conjunto de ordenes lógicas que previamente programadas responden a una secuencia significativa. De acuerdo aTarifa, E. (2005), el evaluador "es el conjunto de procedimientos que procesarán el modelo para obtener los resultados de la simulación. Puede 
contener rutinas para la resolución de sistemas de ecuaciones, generadores de números aleatorios, rutinas estadísticas, etc." (p. 4).

3. La interfaz: es el entorno diseñado para que el operario interactué con el simulador, de tal forma que permite la manipulación de algunas variables con relación al sistema simulado. En la actualidad, y gracias a los adelantos en materia de nuevas tecnologías, es posible diseñar interfaces ergonómicas que posibilitan a los aprendices una interacción con el material real. Por ejemplo, las cabinas utilizadas para la simulación de vuelos permiten en tiempo real, operar, maniobrar y observar el resultado del sistema sobre la acción ejecutada del operario, con las mismas características que presenta la cabina de un avión real.

Con relación a lo expuesto hasta este momento, cabe resaltar que existen simuladores que no se especializan en representar la realidad, este es el caso de simuladores que en forma gráfica muestran un comportamiento con relación a algunas variables que intervienen, pero no muestran la realidad propiamente dicha. Por ejemplo, la curva generada mediante un gráfico con relación a la edad y ritmo cardiaco de un individuo. Estos simuladores interpretan los valores de las variables edad y ritmo cardiaco, para luego mostrar una gráfica que es representativa de la realidad, pero no simulan la realidad propiamente dicha, es decir, no muestran al paciente y sus consecuencias.

En algunos modelos de simulación, la reproducción de la realidad no es necesaria, sin embargo, sí lo es, la relación que ésta tiene con un evento real; mientras que en otras situaciones la reproducción fiel de la realidad resulta siendo mucho más importante. Según Martí, E., la fidelidad de la simulación está en dependencia de los objetivos propuestos.

"En determinadas circunstancias la fidelidad entre el modelo y la realizada es de gran importancia. Pensemos por ejemplo, en las posibilidades de instrucción de pilotos o de expertos en el funcionamiento de cualquier maquina compleja; o pensemos también en la preescisión de ciertos acontecimientos climáticos. La utilización de un modelo que reproduce con fidelidad los parámetros y relaciones fundamentales de la situación real es, en estos casos, imprescindible. Pero nos puede interesar, por ejemplo, presentar a los alumnos ciertos parámetros para que sean ellos quienes construyan un modelo casual con el fin de resolver determinados problemas: problemas científicos, problemas ligados a la evolución de la temperatura de un cuerpo que se enfría (Vitale, 1991b), etc. En este caso el énfasis está puesto en el proceso mismo de modelación y no en el de exploración de una situación de simulación” (Martí, E., 1992. p. 189).

\section{Potencialidades pedagógicas de los entornos de simulación}

Revisando los aportes experimentales que sobre el uso de las simulaciones en el sector educativo se han desarrollado, es claro percibir que los entornos de simulación son recursos que presentan diversos aportes a este sector. Sin embargo, la efectividad de las herramientas utilizadas en el proceso de enseñanza y aprendizaje no depende sólo de los artefactos, sino que existen muchos otros factores que influyen directamente, tales como: estilos, ritmos, preferencias, capacidades, estrategias de aprendizaje y antecedentes historiales del aprendiz con relación a los conocimientos previos.

Por tanto, "el aprendizaje depende esencialmente del ser que aprende, no de forma directa de lo que desea o se propone el que enseña" (Gimeno Sacristán, J. y Pérez 1992). Desde esta posición, se hace necesario reconocer en los contextos un elemento potenciador de las motivaciones que conllevan a generar aprendizaje. Esto es, el entorno educativo adecuadamente prediseñado, potencia las posibilidades de los individuos para la construcción del conocimiento, pues, el factor pedagógico de los entornos depende en gran medida de la metodología empleada.

Si metodología es "la ciencia que estudia los métodos, técnicas, procedimientos y medios dirigidos a la enseñanza de una disciplina dada"; las tecnologías deben ser vistas como herramientas para instrumentar los métodos y no como métodos propiamente dichos (Arias, L. 2004). 
El uso de elementos tecnológicos, para nuestro caso, los simuladores computarizados, debe ser visto como un recurso que media y faculta a los aprendices en la construcción del conocimiento. Así que las simulaciones computarizadas deben ser vistas como recursos técnicos de orden material, que le facilitan al educando la interacción, estudio, y/o modelación de la realidad o de una parte de esta. Desde esta perspectiva, "los ordenadores personales constituyen medios técnicos de especial significación para el contexto metodológico actual" y no elementos pedagógicos por sí solos. En definitiva, el valor pedagógico y didáctico de los medios info-virtuales, es proporcionado por el contexto metodológico en el que se explotan sus cualidades.

Ahora bien, si la simulación es vista como un recurso que facilita la ejecución de metodologías educativas $y$, por consiguiente, de procedimientos de aprendizaje, entendiendo estos como el conjunto de acciones ordenadas, orientadas a la consecución de una meta u objetivo ¿por qué no se deben clasificar como metodología propiamente dicha?

Para que la simulación se pueda clasificar como una metodología, tendría que ser por sí sola un elemento suficiente para penetrar en la esencia de la realidad, y esto no es así, pues la modelación de un evento real no es un hecho suficiente para que se produzcan actividades de interacción significativa. Desde aquí se hace necesario el empleo de procesos, procedimientos y métodos [2] que en forma secuencial ayuden a la interpretación de esa realidad. Por otro lado, y desde un enfoque sociocultural del aprendizaje, las simulaciones por si solas no pueden generar eventos e interacciones sociales o sistemas colaborativos. Es cierto que sí generan un entorno común de significados, pero en dependencia de un proceso metodológico que induce a la reflexión. En consecuencia, se deben reconocer las simulaciones como elementos que coaccionan con la metodología en el proceso de enseñanza y aprendizaje. Así pues, las simulaciones son instrumentos del método que corresponden a una parte de las actividades que comprenden un diseño metodológico y no son por sí solas metodología de enseñanza y aprendizaje.

Muchos autotes clasifican los entornos de simulación como métodos, es el caso de Arias, L. (2004), quien al respecto señala:

"Visto desde el proceso de enseñanza, el procedimiento es un conjunto de acciones del profesor encaminadas al logro de un objetivo, que jerárquicamente se deriva del que orienta al método que se emplea para dirigir el aprendizaje de los estudiantes, y que tienen lugar por medio del conjunto de experiencias de naturaleza cognitiva e instrumental que ha acumulado, así como de los

correspondientes soportes técnicos. Desde el aprendizaje, el procedimiento constituye el conjunto de acciones que desarrolla el estudiante para ir penetrando gradualmente en la realidad que estudia, y la consiguiente construcción de un sistema cognitivo-instrumental que le permitirá seguir penetrando en ella.

La simulación, por tanto, se constituye en procedimiento, tanto para la formación de conceptos y construcción en general de conocimientos, como para la aplicación de éstos a nuevos contextos a los que, por diversas razones, el estudiante no puede acceder desde el contexto metodológico donde se desarrolla su aprendizaje".

En definitiva y sin infravalorar las potencialidades implícitas en los entornos de simulación generados por ordenador, estos carecen de valor didáctico fuera de un contexto metodológico. Por lo tanto, si contamos con un adecuado diseño metodológico, las simulaciones se pueden percibir como una pertinente alternativa dentro de la necesidad de encontrar una solución a las descontextualizadas actividades educativas al interior del aula de clase.

\section{Entornos de simulación computarizada, espacios de realidad que generan actividad.}

Partiendo de la premisa que sustenta que "los contextos situacionales de actividad no se hallan determinados por el contexto físico, es más, son creados por participantes en el contexto" (Wertsch, J. 1988, p.223), se puede deducir que la actividad generada por los individuos, depende en gran medida 
de las circunstancias situacionales que confiere la interacción con la realidad. Ahora bien, es pertinente resaltar que "las acciones que componen una actividad no son sólo ejecuciones de tipo práctico, sino también de tipo sensorial, representativo, conceptual, lingüístico o afectivo-emocional. Generalmente son la combinación interfuncional de todas ellas”(Molina, L., 1997, p.117).

En relación con los programas de simulación, estos pueden proveer un eje de conexión entre el conocimiento y el mundo. Por esto, la actividad de los individuos se genera en la interacción con el mundo y las relaciones causales de esta interacción en respuesta a un evento simulado de la realidad.

Otro punto que se debe tener presente, es que los contextos físicos también modifican la acción de los actores en la actividad. Para este caso, los simuladores computarizados facultan un determinado tipo de posibilidades que permiten su diseño y manipulación a voluntad; permitiendo así que los usuarios generen actividad controlada dentro de unos fines particulares. Este aspecto se torna en un elemento positivo para nuestro caso, pues posibilita que la actividad educativa sea premeditada y planeada para que el estudiante responda de una manera determinada en relación con la situación y las posibilidades que el contexto le presenta. Con esto no se dice que el simulador deba ser un plan secuencial que lleve de la mano al aprendiz, sino más bien debe ser un entorno de oportunidades situacionales diseñadas para posibilitar el fin, que es educar.

Esta característica permite diseñar modelos de instrucción dentro de la forma natural como las personas aprenden, pues, como se ha demostrado en variadas investigaciones, "las personas inexpertas no actúan según planes (ni siquiera cuando habían leído las instrucciones), sino sobre la base de conjuntos de destrezas previamente incorporadas que habían ido configurando en una trayectoria histórica de acciones similares" (Schuman, L. Citado en Streibel, M. 1989, pp. 215-234).

Desde lo anterior, no es concebible interpretar los entorno de simulación como un simple plan de instrucción, los entornos de simulación deben ser vistos como elementos de realidad que posibilitan el desarrollo de los contextos de actividad e interacción significativa. Esta perspectiva permite la construcción del conocimiento desde la misma interacción con el contexto, donde el "conocimiento supone una experiencia específica y contextual [donde] nuestras acciones se producen no desde planes construidos racionalmente, sino sobre la base de nuestras propias destrezas incorporadas, que son sensibles al contexto" (Ibidem).

En definitiva, la actividad al marco de los entornos de simulación computarizada, "ha de verse como la interacción con los artefactos e instrumentos bajo las circunstancias sociales que los envuelven y no sólo como interacción entre sujetos sociales (...). Los recursos e instrumentos, sobre todo los objetos informáticos y automatizados, son también mediadores y actuantes entre el mundo y los agentes"(Lozares, C., 2000, pp. 97-131). Asimismo, la actividad vista como la mediación entre contexto situacional, individuos y el medio circundante, exige unos elementos mínimos para su desarrollo, Por lo tanto, un diseño metodológico apoyado con entornos de simulación como contextos de actividad, debe por lo menos posibilitar los siguientes elementos:

$\S$ La percepción e interacción de y con la situación.

$\S$ Inmersión.

$\S$ Participación.

$\S$ Colaboración.

$\S$ Aprehensión de los contenidos como "cualidades perceptivas de los objetos, materiales y procesos que componen la actividad; las relaciones especiales, analógicas, casuales, asociativas o categoriales que mantienen con otros elementos de la realidad" (Molina, L., 1997, p.167).

$\S \quad$ El compromiso del aprendiz en la situación. 
$\S$ El desarrollo de las acciones y operaciones que pueden ayudar a culminar la tarea.

Con relación al hecho de que la actividad es un producto de la interacción del hombre con su entorno, la realidad simulada se torna en una oportunidad para que los individuos ajusten los instrumentos psicológicos y conceptuales a los requerimientos situacionales. Es decir, estos entornos potencian la mediación instrumental socialmente situada, convirtiéndose en el punto de apoyo para la generación de actividad consciente y significativa. En palabras de Vygotsky, la formación de los procesos psicológicos y el pensamiento conciente de los hombres, es un evento socialmente situado en relación con los acontecimientos que su entorno le impone (Vygotsky, L. 1979). Visto de esta manera, los entornos de simulación computarizada -apoyados con un adecuado diseño metodológico-, proporcionan al aprendiz un contexto variado de posibilidades situacionales que motivan a generar actividad significativa.

Aquí las herramientas psicológicas se ponen a prueba mediante una exigente adaptación y ajuste a las condiciones situacionales de la realidad simulada. Es pertinente resaltar que las affordances que el entorno pedagógico le debe proporcionar al individuo, tienen que ser muy ricas, convirtiéndolo así en un contexto de creación, interacción, uso y aplicación de herramientas y, por consiguiente, motor que genera pensamiento consciente. Asimismo, y con ya se ha dicho, no se puede confundir esto con proporcionar elementos construidos como piezas de rompecabezas para que el aprendiz les junte camino a culminar la actividad. Lo que debe proporcionar el contexto son las herramientas de mediación, no la solución, es decir, elementos que permitan al sujeto interpretar la situación y aprehender las oportunidades.

En análisis realizado por Nardi, B. (2001), se resalta el valor de una actividad, así como la reciprocidad que existe entre los niveles presentados por Leontiev, A. (actividad, acciones, operaciones [3] ), y los elementos que integran la construcción de un pensamiento consciente. Esto es, las situaciones generan actividad, estas a su vez genera acciones que se materializan en operaciones, pero estas últimas en dependencia de las circunstancias, generan nuevas acciones y nuevas actividades, y así sucesivamente.

Si la actividad se desarrolla al marco de eventos reales y significativos, resulta siendo un evento altamente consciente.

"Las acciones consisten en cadenas de las operaciones, que son rutinas habituales bien definidas, usadas como respuestas a las condiciones generadas durante la ejecución de la acción. Cada operación es inicialmente una acción consciente, consistiendo las fases de la orientación y de la ejecución, pero cuando el modelo correspondiente es bastante bueno y la acción se ha practicado bastante tiempo, la orientación de la fase estará en decadencia y la acción concurrirá en una operación, que es mucho más fluida. Al poco tiempo, se crea una nueva acción que tendrá un alcance más amplio y contendrá la nueva operación, recientemente formada como una sub-parte. Por otro lado, cuando las condiciones cambian, se genera la operación y vuelve otra vez al nivel de la acción consciente (de modo que no es un reflejo condicionado)".p. 29.(Traducción propia).

En el contacto de los individuos con un contexto de realidad, a diferencia de la interacción que se desarrolla en un entorno descontextualizado, "las actividades están cambiando y se están convirtiendo siempre. El desarrollo está ocurriendo en todos los niveles; las nuevas operaciones se forman de acciones anteriores mientras que las habilidades de los participantes aumentan; correspondientemente, en el nivel de acciones el alcance de nuevas acciones se está inventando y a la vez se están incrementando, se están experimentando y se están adaptando como respuestas a las nuevas situaciones o posibilidades encontradas en el proceso de transformar el objeto." (Nardi, B., 2001, p. 33). (Traducción propia).

Por otro lado, cabe resaltar que el desarrollo de la actividad está necesariamente mediado por los artefactos, esto es, los artefactos son mediadores del pensamiento y del comportamiento humano; mas no ocupan el mismo espacio ontológico. Sin embargo, los artefactos al igual que las herramientas 
sicológicas se especializan y desarrollan en la interacción individuo-entorno, de modo que la actividad como un proceso consciente, permite generar construcciones significativas a toda escala. Así pues, si visualizamos los entornos de simulación como artefactos que posibilitan la mediación del individuo con su entorno, podemos ver en dicha relación, una oportunidad para ajustar la actividad y los elementos que la conforman y a la especificidad situacional. De modo que en este caso, la actividad estará condicionada por las posibilidades artefactuales y la relación reglada de estas posibilidades con el entorno.

Otro elemento primordial dentro de un sistema de actividad, es el contexto cultural. La cultura forma parte de un marco socialmente establecido, hecho que da estructura a lo que piensa, lo que dice y lo que hace. Así pues, desde los sistemas de actividad, la condición de miembro de una comunidad establece la forma como se perciben los acontecimientos en dependencia de la interpretación social y cultural. En tal sentido, el individuo sitúa su comportamiento como miembro de esa comunidad, adaptándose activamente a la condición cambiante de la situación. Al respecto, la teoría socio-cultural ha caracterizado la cultura como un legado de artefactos destinados a mediar la conducta del hombre frente a su entorno situacional.

Por lo tanto, si se evalúa la interacción con el entorno de simulación desde sus posibilidades o limitaciones con relación a la interpretación cultural, podemos entortar dos estadios:

Primero, si estos entornos son vistos como artefactos de mediación dentro de la actividad, la interpretación que se genera está en total dependencia del contexto sociocultural y no de ellos mismos, es decir, el valor y la interpretación de dicho artefacto depende del contexto cultural en el que es empleado.

Segundo, si estos espacios se consideran entornos de situación, desde las posibilidades operativas que ellos facultan, dicha situación está condicionada por el comportamiento real del sistema y por la interpretación que le imprime el contexto cultural. Por lo tanto el simulador no condiciona su interpretación, ésta sigue siendo cultural, además que dependerá de las herramientas conceptuales que trae el aprendiz y no propiamente de la simulación.

Desde lo anterior, es pertinente resaltar que esta apreciación está condicionada por las posibilidades representativas de dicho entorno con relación a la realidad. Por lo tanto, si los simuladores computarizados responden a normas y reglas naturales, su interpretación parte del sujeto en relación con la interpretación de dicha realidad -ya sea cultural, situacional, social o de actividad-.

Ahora bien, partiendo de la premisa que sustenta que "la mediación cultural y el hecho de que el pensamiento se funde en la actividad implican la especificidad contextual de los procesos mentales" (Moll, L. 1990, p. 112), es de sospechar que los entornos de simulación no coartan dicha especificidad, pues la actividad emergente no se repite aunque los eventos a simular sean los mismos. Esto se torna en una característica positiva a la hora reciclar el material dentro de la instrucción, pues la variedad de experiencias está enmarcada dentro de las posibilidades que el diseño metodológico le imprime.

En conclusión, los entornos de simulación no impiden el flujo natural de la actividad, así como tampoco imponen un repertorio de interpretaciones socio-culturalmente establecidas; por el contrario, solo responden a unas reglas naturales de comportamiento con relación a una situación. De modo que, se puede a firmar que estos entornos cobran significado en dependencia de la actividad y del grupo socio-cultural en el que son empleados.

Otra característica que hace de los entornos de simulación una herramienta adecuada para ser integrada al proceso de enseñanza y aprendizaje, es que estos espacios no coartan ni condicionan la interpretación de la situación ni la interacción de los actores dentro de la actividad. Por otro lado, la aprehensión del significado, del contenido, y por consiguiente del aprendizaje, no es impuesta por el software o por el profesor, estos elementos son construidos con relación a la particularidad de dicho 
evento situacional que se genera de la interacción con la simulación.

Bibliografía

AGUADED, J. \& CABERO, J. (2002) Educar en red: Internet como recurso para la educación. Málaga, Aljibe.

AKPAN, J. (2001) Issues Associated with Inserting Computer Simulations into Biology Instruction: A Review of the Literature. electronic journal of science education Vol 5 (3) http://unr.edu/homepage/crowther/ejse/akpan.html.

ALONSO, C. \& GALLEGO, D. (2002) Tecnologías de la información y la comunicación, Revista de Educación, 329, 181-201.

BANDERA, J. (2002) La globalización y sus rostros, Cuadernos de Realidades sociales, 59-60, 5-9.

BARRIO, J.; CLIMENT, R.; JARDÓN, P.; SOLER, M. \& VILLAVERDE, V. (1998) Arqueología, hominización, sociedades cazadoras - recolectoras: Orientación teórica y praxis didáctica. Valencia, Libres.

BARTOLOMÉ, A. (1989) Nuevas tecnologías y enseñanza. Barcelona, Graó.

BETTETINI, G. \& COLOMBO, F. (1995) Las nuevas tecnologías de la comunicación (1ª edición). Barcelona, Paidós.

BLÁZQUEZ, F.; CABERO, J. \& LOSCERTALES, F. (1993) Nuevas tecnologías de la información y comunicación para la educación. Sevilla, Alfar.

BORRAS, I. (1999) Enseñanza y aprendizaje en la Internet: una aproximación crítica. http//www.didácticahistoria.com/tecedu/tecedud12htm

BROFENBRERNNER, U. (1987) La ecología del desarrollo humano. Barcelona, Paidós.

BROWN, J.; COLLINS, A. \& DUGUID, P. (1989) Situated Cognition and the culture of learning, Educational Researcher,18(1), 33-42.

BRUNNER, J. (1984) Acción, pensamiento y lenguaje. Madrid, Alianza Editores.

BURBULES, N. \& TORRES, C. (2001) Globalización y educación, Revista de Educación, número extraordinario, 13-29.

CABERO, J.; BARTOLOMÉ, A.; CEBRIÁN, M.; DUARTE, A.; MARTÍNEZ, F. \& SALINAS, J. (1999) Tecnología Educativa. Madrid, Síntesis.

CARRADA, G. (2000) La prehistoria del hombre: del origen al neolítico. Madrid, Editex.

CASTELLS, M. (2001) Materiales para una teoría preliminar sobre la sociedad de redes, Revista de Educación, número extraordinario, 41-58.

CASTILlEJO, J.; COLOM, A.; ESCÁMEZ, J.; GARCÍA, J.; SANVISENS, A.; SARRAMONA, J. \& VÁZQUEZ, G. (1986) Tecnología y educación. Barcelona, Ceac.

CEBRIÁN, M. (1983) Teoría y técnica de al información audiovisual. Madrid, Alambra S. A.

CHAIKLIN, S. \& LAVE, J. (2001) Estudiar las prácticas. Argentina, Amorrortu. 
CLANCEY, W. (1995) A tutorial on situated learning. Proceedings of the International Conference on Computers and Education (Taiwan) Charlottesville, 49-70, http://www.cogprints.ecs.soton.ac.uk/archive/00000323/00/139.htm. (consultado 18-04-2004).

COLE, M. (1999) Psicología Cultural. Madrid, Morata.

COLL C.; PALACIOS J.; MARCHESI, A. (1990) Desarrollo psicológico y educación, II sicología de la educación. Madrid, Alianza Psicológica.

COLORADO, A. (1997) Hipercultura visual. El reto hipermedia en el arte y la ecuación. Madrid, Editorial Computense.

CORRALES, J.; GARCÍA, L.; HIYLES, C.; MARTÍ, E.; MENDELSOHN, P.; OSÍN, L.; REQUENA, A.; VERGÉS, M. y VITALE, B. (1988) Tecnología y educación. Madrid, Narcea.

DELVAL, J. (1986) Niños y maquinas. Los ordenadores y la educación. Madrid, Alianza.

DE PABLOS, J. \& SEGURA, J. (1998) Nuevas tecnologías: comunicación audiovisual y educación. Barcelona, Cedecs.

DEWEY, J. (1989) Cómo pensamos: nueva exposición de la relación entre pensamiento reflexivo y proceso educativo. Barcelona, Paidós.

DÍAZ, B. (2003) Cognición situada y estrategias para el aprendizaje colaborativo, Revista Electrónica de Comunicación, 5 (2), 12. http://www.redie.ens.uabc.mx/voL5no2/contenido-arceo.html. (consultado 10-03-2004).

DURSTELER, J. ( 2002) Habilidades: La perspectiva ecológica de Gibson, Revista Digital de Infovis. net, mensaje, 72, 1. http//www.infovis.net/revista/2002/num_72html. (consultado 15-11-2003).

GARCÍA CARRASCO, J. \& GARCÍA DEL DUJO, Á. (2001) Teoría de la Educación II. Salamanca, Ediciones Universidad de Salamanca.

GARCÍA DEL DUJO, Á. \& MARTÍN GARCÍA, A. V. (2002) Características pedagógicas de los entornos virtuales de aprendizaje, Teoría de la educación. Revista Interuniversitaria, 14, 67-92.

GARCÍA-VALCÁRCEL, A.; (2003) Tecnología educativa. Implicaciones educativas del desarrollo tecnológico. Madrid, La muralla.

GARCÍA, C. \& VILLATORO, F. (2004) técnicas de simulación mediante el método montecarlo. ttp://polaris.lcc.uma.es/ villa/mmtc/tema14.pdf.

GARRIDO, A. (2003) El aprendizaje como identidad de participación en la práctica de una comunidad virtual. http://www.uoc.edu/in3/dt/20088/index.html. (18-02-2004).

GERSTEN, R. \& BAKER, S. (1998) Real world use of cientific concepts: integrating situated cognition with explicit instruction, Exceptinal Children, 65(1), 23-36.

GROS, B. (2002) El ordenador invisible: Hacia la aproximación del ordenador en la enseñanza. Barcelona, Gedisa.

HABERMAS, J. (1986) Ciencia y tecnología como ideología. Madrid, Técnos.

HILERA, J.; OTÓN, S. y MARTÍNEZ, J. (2000) Aplicación de la Realidad Virtual en la enseñanza a través de Internet. http://www.ucm.es/info/multidoc/multidoc/revista/num8/hilera-oton.html (04-082004). 
HUNG, D. (2002) Situated cognition and problem-based learnig: implications for learning and instructions with Tecnology, Journal of interactive Learnig research, 13(4), 393-414.

Jonson, L. \& Rickel, J. (1997) Steve: An animaated Pedagogical Agent for peocedural Training in virtual environments, Sigart Bulletin, 16-21.

KILMAN, D. (1993) Technology and situated cognition, Tecnology \& learnig, 14(1), 86(1).

LACASA, P. (1994) Aprender en la escuela, aprender en la calle. Madrid, Visor.

LACASA, P. (1995) Los deberes escolares ¿entre la certeza y la duda?. www.uco.es/ 'ed1ladip/investiga/debint.htm. (18-12-2003).

LAVE, J. \& WENGER, E. (1991) Situated Learnig Legitimate Peripheral Participation. USA, Cambridge University Press.

LEARNING TECHNOLOGY CENTER, (1992) The Aventures of Jasper Woodbury.http://peabody.vanderbilt.edu/projects/funded/jasper/Jasperhome.htm (20-06-2004).

LEONTIEV, A.; LURIA, A. \& VYGOTSKY, L. (1973) Psicología y pedagogía. Madrid, Akal.

LEONTIEV, A. (1983) El desarrollo del psiquismo. Madrid, Akal editores.

LOZARES, C. (2000) La actividad situada y/o el conocimiento socialmente distribuido. http://www.bib.uab.es/pub/papers/02102862n62p97.pdf.

LURIA, A. (1984) Conciencia y lenguaje. Madrid, Visor.

MAJÓ, J. \& MARQUÉS, P. (2002) La revolución educativa en la era Internet. Barcelona, Praxis.

MARCHESI, A. (1990) Desarrollo psicológico y educación II. Madrid, Alianza.

MARTÍ, E. (1992) Cuadernos de educación. Aprender con ordenadores en la escuela. Barcelona, IceHorsori.

MARTÍNEZ, S. \& Requena, A. (1986 Dinámica de sistemas. Simulación por ordenador. Madrid, Alianza.

McLELLAN, H. (1994) Situated Learning: Continuing the Conversation, Educational Technology, Octubre, 7-8.

MIRABITO, M. (1998) Las nuevas tecnologías de la comunicación. Barcelona, Gedisa.

MOLINA, L. (1997) Participar en contextos de aprendizaje y desarrollo. Barcelona, Paidós.

MOLL, L. (1990) Vygotsky y la educación. Argentina, Aique.

NARDI, B. (2001) context and consciousness. Activity theory and human-computer interaction. London, Mit Press.

ORTEGA, M. \& BRAVO, J. (2001) Sistemas de interacción persona- computador. España, Ediciones Universidad de Castilla La Mancha.

POZO, J. (1989) Teorías cognitivas del aprendizaje. Madrid, Morata.

QUINTANILLA, M. A. (1989) Tecnología: Un enfoque filosófico. Madrid, Fundesco. 
RAMÍREZ, L. (2004) La dimensión social de la globalización. Periódico nuevo enfoque, edición de julio, segunda quincena. http://www.libros.com.sv/edicion35/dimencion .html

RIVIÉRE, A. (1985) La psicología de Vygotsky. Madrid, Visor.

RODRIGO, M. (1994) Contexto y desarrollo social. Madrid, Sistemas.

RODRÍGUEZ, O. (2002) Hacia un proyecto renovador del significado del hombre educado: cognición y tecnología. http://www.razonypalabra.org.mx/anteriores/n24/24-orgz.html. (20-02-2004).

RODRÍGUEZ, M. \& GARCÍA, M. (2003) El aprendizaje como problema actual de la educación, Revista Cubana de Investigación, 20 (2), 136-144.

ROGOFF, B. (1993) Aprendices del pensamiento. Barcelona, Paidós.

ROMO, A. (1999) El enfoque sociocultural del aprendizaje de Vygotsky.

http://www.monografias.com/trabajos10/gotsky/gotsky.shtml. (06-10-2004).

SALOMON, G. (1993) Cogniciones distribuidas: consideraciones psicológicas y educativas. Buenos Aires, Amorrortu.

SANCHO, J. (1994) Para una tecnología educativa, Barcelona, Horsori.

SHAO, Y. (1999) The development of an exploratory simulation for situated learning, en: http://www.pals.iastate.edu/simulations/library/papers/mountainsimdevelopment.html. (22-07-2004).

SOLER, E. (2002) Aprendizaje situado: una discusión abierta, Revista de investigaciones educativas, 23(2), 1-22.

STEIN, D. (1998) Situated Learning in Adult Education. Resumen entregado por la base de datos Eric Digest No 195. http://www.ericfacility.net/databases/eric_digests/ed418250.html. (10-01-2004).

STREIBEL, M. (1989) Diseño instructivo y aprendizaje situado: ¿es posible un maridaje?, Revista de Educación, 289, 215-234.

SUÁREZ, C. (2002) Entornos virtuales de aprendizaje: Interfaz de aprendizaje colaborativo. Memoria de Grado, Facultad de Educación, Universidad de Salamanca, Salamanca, España.

TARIFA, E. (2005) Teoría de Modelos y Simulación. Introducción a la simulación. En doc. Virtual, Facultad de Ingeniería - Universidad Nacional de Jujuy 05/05/05

http://www.modeladoeningenieria.edu.ar/unj/tms/apuntes/cp1.pdf.

TOURIÑÁN, J. (2001) Tecnología digital y sistema educativo: El reto de la globalización, Revista de Educación, Número extraordinario, 217-230.

VÁZQUEZ, G. (1986) Tecnología y educación. Barcelona, Ceac.

VYGOTSKY, L. (1979) El desarrollo de los procesos psicológicos superiores. Barcelona, Crítica.

VYGOTSKY, L. (1984) Obras escogidas IV. Moscú, Visor.

WENGER, E. (2001) Comunidades de Práctica. Barcelona, Paidós.

WERTSCH, J. (1988) Vygotsky y la formación social de la mente. Barcelona, Paidós.

WERTSCH, J. (1993) Voces de la mente. Madrid, Visor. 
Notas:

[1] La psicología ecológica concibe la persona desarrollándose en dependencia del ambiente, estudia la forma como el individuo "percibe el ambiente y se relaciona con él”. BROFENBRERNNER, U. (1987) La ecología del desarrollo humano. Barcelona, Paidós, p. 23.

[2] Proceso: desarrollo secuencial de actividades que ejecuta el aprendiz a manera de herramientas que le permiten adentrarse en la realidad objeto de estudio. Método: "Modo de decir o hacer con orden una cosa". "Procedimiento que se sigue en las ciencias para hallar la verdad y enseñarla". (Diccionario de la Real Academia de la lengua Española, 2005).

[3] Véase, LEONTIEV, A. (1983) El desarrollo del psiquismo. Madrid, Akal Editores.

(C) Ediciones Universidad de Salamanca 